\title{
Ciclo financeiro global e liberalização financeira externa: desdobramentos sobre o desempenho macroeconômico brasileiro entre 1995 e $2014^{*}$
}

\author{
Global financial cycle and external financial liberalization: effects over the Brazilian \\ macroeconomic performance in the period 1995-2014
}

\author{
André Moreira Cunha ${ }^{\dagger}$ \\ Pedro Perfeito da Silva ${ }^{\ddagger}$ \\ Mateus Ramalho Ribeiro da Fonseca ${ }^{\S}$
}

\begin{abstract}
Resumo
O artigo avalia o impacto da liberalização financeira externa - nas dimensões de jure e de facto - sobre o desempenho macroeconômico brasileiro, e traz como contribuição potencialmente original o emprego do modelo econométrico de Vetores Autorregressivos com Mudanças de Regime a partir de Correntes Markovianas (MS-VAR). Isso possibilita o enfrentamento da não linearidade dos parâmetros e a estimação de regimes que representam os movimentos do ciclo financeiro global. Quanto a resultados, um choque positivo no grau de liberalização, em ambos os regimes e dimensões, tende a gerar desdobramentos problemáticos em variáveis como risco-país, volatilidade cambial, taxa de juros e nível de atividade.
\end{abstract}

Palavras-Chave: Liberalização financeira; Desempenho macroeconômico; Modelos MS-VAR.

\begin{abstract}
The paper evaluates the impact of external financial liberalization - considering de jure and de facto dimensions -on the Brazilian macroeconomic performance, and it brings as potentially original contribution the use of Markov Switching Vector Autoregressive Model (MS-VAR). It permits combating the nonlinearity of the parameters and estimating regimes which represents the movements of global financial cycle. In terms of results, a positive shock in the degree of liberalization, in both dimensions and regimes, tends to generate problematic developments on country risk, exchange rate volatility, interest rate and level of economic activity.
\end{abstract}

Keywords: Financial liberalization; Macroeconomic performance; MS-VAR models.

JEL Classification: F32; E44; 016.

\footnotetext{
* Agradecemos ao CNPq pelo apoio financeiro e aos(às) pareceristas anônimos(as) pelas sugestões. Os erros e as omissões remanescentes são de nossa inteira responsabilidade.

+ Professor Associado do Departamento de Economia e Relações Internacionais da Universidade Federal do Rio Grande do Sul. Pesquisador do CNPq. Email: andre.cunha@ufrgs.br

‡ Doutorando no Programa de Pós-Graduação em Economia da Universidade Federal do Rio Grande do Sul. Email: pperfeitodasilva@gmail.com

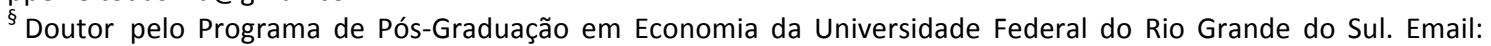
mateusramalho88@hotmail.com
} 


\section{Introdução}

A tendência à liberalização econômica em geral, e à liberalização financeira, em particular, tem caracterizado a economia internacional desde meados dos anos 1980 . Neste período, reemerge um mercado financeiro globalizado, o qual passou a facilitar a movimentação financeira externa, com fluxos muito mais robustos e, ao mesmo tempo, voláteis do que no passado. De fato, a globalização financeira caracteriza-se pela recorrência de crises, como a recente ruptura originada no mercado hipotecário dos EUA e logo alçada à condição de crise global (Arestis et al., 2011; Cynamon et al., 2013). Frente a tal cenário, a política monetária expansionista e não-convencional dos Estados Unidos (EUA) fortaleceu a discussão acerca do ciclo financeiro global e seus desdobramentos nos países que não possuem moeda conversível. Esses são, tipicamente, cycle takers, com suas dinâmicas macroeconômicas domésticas sendo condicionadas, de forma decisiva, pelo ciclo determinando no centro do sistema monetário e financeiro internacional (Ocampo et al., 2007; Paula et al., 2017).

Considerando tais aspectos, o presente artigo avalia os impactos macroeconômicos da liberalização financeira externa (LFE) sobre a economia brasileira no período 1995-2014, que coincide com a estabilização monetária pós-Real e com o aprofundamento da dinâmica de integração com instabilidade. Assim, busca-se contribuir com a literatura que se dedicou ao caso do Brasil': (i) com a incorporação da relação entre o grau de liberalização financeira externa da economia brasileira com o ciclo financeiro global (Borio, 2012; BIS; 2014, 2015), medido por meio do VIX, em linha com as sugestões de trabalhos como os de Rey (2013), Nier et al. (2014), IMF (2017), Coimbra e Rey (2017), Cerutti et al. (2017), dentre outros; e (ii) pela utilização do modelo econométrico de Vetores Autorregressivos com Mudanças Markovianas (MS-VAR) para avaliar os efeitos da LFE sobre o desempenho macroeconômico brasileiro. Tal metodologia se mostra adequada ao tratamento das não linearidades típicas dos movimentos cíclicos dos fluxos de capitais. Adicionalmente, o artigo amplia o horizonte temporal de análise com respeito aos trabalhos anteriores, particularmente os inspirados na tradição póskeynesiana (ver seção 2), que se debruçaram sobre esse tema. Tal esforço de pesquisa é convergente com trabalhos prévios no sentido mais geral de se buscar evidências sobre o grau de liberalização e de integração financeira externa no Brasil e de como tal dinâmica tem afetado a nossa economia.

Além desse objetivo principal, enfrentado na quarta seção, o artigo apresenta, respectivamente, nas próximas duas seções: uma revisão da literatura teórica e empírica e uma breve exposição da história do processo brasileiro de liberalização financeira. Parte-se da hipótese de que o grau de liberalização financeira da economia brasileira responde aos movimentos do ciclo financeiro global e que um avanço liberalizante tem desdobramentos macroeconômicos potencialmente problemáticos.

\footnotetext{
${ }^{1}$ Ver, dentre outros, Arida (2003a, 2003b, 2004), Belluzzo e Carneiro (2004), Ferrari Filho et al. (2005), Faria et al. (2009), Paula (2011) e Paula et al. (2012).
} 


\section{Liberalização financeira externa: debate teórico e empírico}

Em termos históricos, Minsky (1993) conecta a expansão do comércio internacional no pós-guerra com o avanço da integração entre economias e sistemas financeiros ao redor do mundo, engendrando a centralidade das empresas multinacionais e das decisões de portfolio dos intermediários financeiros. Tais desenvolvimentos estão na origem da ruptura da ordem de Bretton Woods, substituída pela dominância de regimes cambiais flexíveis, especialmente quando se considera o caso das economias avançadas, e pelo avanço dos desequilíbrios nos balanços de pagamentos, ajustados via movimentos de capitais de curto prazo. Nesse contexto, segundo Prasad et al. (2003), o conceito de liberalização financeira externa possui duas dimensões: i) a liberalização de jure ou abertura financeira, a qual advém de mudanças legais e/ou regulatórias que promovem a redução das restrições aos movimentos de capital; e ii) a liberalização de facto ou integração financeira, que pode ser observada tanto a partir da crescente sincronização de preços e retornos nos mercados doméstico e global quanto por meio da expansão do montante de fluxos ou estoques que entram e saem da economia nacional.

Os entusiastas da LFE associam esse processo à ampliação no nível de atividades e a atenuação do ciclo econômico doméstico. Sugere-se que o acesso ampliado à poupança externa permitiria a elevação dos investimentos e, com isso, da taxa de crescimento nas economias receptoras de capitais. Do ponto de vista dos países de origem daqueles recursos, o atrativo da integração aos mercados financeiros internacionais adviria da possibilidade de diversificar riscos e ampliar a remuneração da sua poupança. Por assumir que os capitais privados fluem pro-ciclicamente, considera-se que os países hospedeiros podem ter naqueles uma fonte de atenuação dos impactos recessivos e de piora do bem-estar pela absorção de recursos forâneos. A lógica desse movimento é singela: em momentos de crise, os preços dos ativos denominados em moeda doméstica caem, ao mesmo tempo em que a taxa de câmbio se eleva. Isso tornaria extremamente atraente o processo de aquisição de ativos locais. Com a entrada de capitais, a recessão é suavizada (Prasad et al., 2003; Obstfeld e Taylor, 2004). Ademais, haveria efeitos colaterais positivos como o desenvolvimento do sistema financeiro doméstico, a evolução institucional, a disciplina sobre a política governamental capazes de ampliar a produtividade total dos fatores. Em termos macroeconômicos, tais avanços culminariam na diminuição da taxa de juros, da volatilidade cambial e do risco-país, o que levaria à elevação da taxa de crescimento do produto² .

Por outro lado, nos termos de Palley (2009), as críticas à liberalização financeira se organizam em torno dos seguintes problemas: (i) perda de autonomia da política monetária doméstica e transmissão de choques externos; (ii) desdobramentos negativos no desempenho macroeconômico; e (iii) impactos sobre o sistema financeiro doméstico. No primeiro ponto, a própria literatura convencional traz o conceito de ciclo financeiro global, já exposto na introdução, o qual se desdobra na amplificação de flutuações econômicas, deslocamentos econômicos e problemas financeiros associados aos desequilíbrios patrimoniais dos agentes, relacionando-se ao comportamento pró-cíclico dos mercados financeiros (Borio, 2012). Por sua vez, Rey (2013), Nier et al. (2014), IMF (2017), Coimbra e Rey (2017), Cerutti et al. (2017),

\footnotetext{
${ }^{2}$ Tal esforço teórico remonta a Balassa (1989), Fischer (1998), Henry (2007), Levine (2001), McKinnon (1973), Mishkin (2005), Shaw (1973), dentre outros.
} 
dentre outros, encontram evidências de que os fluxos de capitais (principalmente, de curto prazo) para economias em desenvolvimento respondem predominantemente a fatores externos a estas, como o sentido da política monetária estadunidense, o apetite frente ao risco dos investidores globais e o desempenho das economias avançadas.

Se a visão recente que emerge na literatura convencional abre espaço para a percepção de que os mercados financeiros apresentam um comportamento desestabilizador e que produz efeitos assimétricos entre os distintos setores econômicos, estratos sociais e tipos de economia, a tradição pós-keynesiana oferece referências teóricas e normativas há muito consolidadas nesses temas (Lavoie, 2014; Davidson, 2015).

Assim, por exemplo, Crotty (1983) sublinha a diferença das agendas liberal e keynesiana acerca da mobilidade de capitais. A primeira prioriza a equalização das taxas de juros ao redor do mundo, enquanto a segunda busca a preservação da autonomia da política monetária doméstica. Nesse sentido, Gallagher (2012) afirma que no período ascendente do ciclo financeiro, os especuladores acessam os mercados emergentes em busca de ganhos com diferenciais de taxa de juros e movimentos na taxa de câmbio (carry-trade), gerando efeitos desestabilizadores em países em desenvolvimento, portadores de sistemas financeiros menos desenvolvidos e diversificados. Portanto, há que se notar que não há identidade necessária entre os interesses dos agentes dominantes do mercado financeiro global e a estabilidade financeira de um país determinado (Kregel, 1996), o que coloca a necessidade de administração da conta capital por meio de controles e políticas macroprudenciais.

Com respeito à conexão entre avanço da LFE e desempenho macroeconômico, a literatura pós-keynesiana enfatiza que os influxos excessivos de capital na fase ascendente do ciclo financeiro global podem engendrar uma perda de competitividade das exportações devido à apreciação cambial (Oreiro, 2004). Na mesma linha, Kregel (2004) responsabiliza a necessidade de atrair capitais estrangeiros em um contexto de liberalização financeira pela manutenção do diferencial de taxa de juros no caso dos países em desenvolvimento. Tal reflexão remonta à Keynes (1976), que argumenta que os fluxos de capitais estrangeiros determinam as condições domésticas e comerciais, muito mais do que o contrário, de modo que se estabelece um conflito entre a atração de capitais e a acomodação do padrão cíclico do investimento doméstico. Problemas associados a tal dinâmica são particularmente mais intensos em países periféricos que não emitem moedas conversíveis internacionalmente (Paula et al., 2017).

Quanto aos impactos de longo prazo, destacam-se diversas contribuições póskeynesianas. Davidson (2015) relaciona a volatilidade cambial, resultante do contexto de liberalização financeira e regime cambial flexível, com o aumento da incerteza dos agentes, os quais reagem diminuindo o horizonte no qual se engajam em compromissos, o que afeta negativamente o ritmo do investimento produtivo e, consequentemente, o crescimento do produto. Thirlwall (2005) observa que a diminuição da competitividade das exportações industriais, devido à apreciação cambial, amplia o hiato entre as elasticidades-renda das exportações de países desenvolvidos e em desenvolvimento.

A literatura convencional reconhece que há impactos potencialmente negativos de medidas liberalizantes sobre o sistema financeiro. Prasad et al. (2007) lembram que o capital 
estrangeiro, ao invés de acelerar a importação de melhores práticas, pode auxiliar na ampliação do poder de mercado de intermediários financeiros oligopolistas domésticos, bem como tomar para si o financiamento dos setores mais dinâmicos. Quanto à precipitação de crises financeiras (bancárias, cambiais ou ambas) em países em desenvolvimento, uma tradição mais próxima ao pensamento econômico dominante sublinha a conexão entre tomada excessiva de risco pelos bancos a partir da rede governamental de segurança (Mishkin, 2005), do distanciamento dos especuladores de curto prazo frente aos fundamentos de longo prazo (Stiglitz, 2000) e das externalidades decorrentes do comportamento especulativo (Korinek, 2011). As interpretações pós-keynesianas reconhecem tais aspectos e avançam ao relacionar as medidas liberalizantes ao fortalecimento do comportamento especulativo (já existente em uma economia monetária de produção), isso afeta negativamente o ritmo de crescimento, afinal, o investimento passa a ser prejudicado pela ascensão dos custos financeiros decorrentes do favorecimento do financiamento especulativo de curto prazo frente ao de longo prazo (Kregel, 1996; Paula et al., 2012; Palley, 2013).

Passando ao debate brasileiro, Arida (2003a, 2003b, 2004) defende a abertura total da conta capital e financeira, assumindo que a manutenção de controles administrativos em transações financeiras com moeda estrangeira levaria a taxas de juros externa e internas mais altas, bem como ampliaria o prêmio de risco. Dessa forma, a conversibilidade plena aumentaria a confiança na política econômica e na capacidade de pagamento da dívida interna e externa, o que possibilitaria um superávit primário menor e uma taxa privada de investimento maior. Além disso, haveria uma diminuição do impacto das flutuações da renda sobre o consumo e uma estabilização dos fluxos de entrada e saída de capitais, reduzindo a amplitude da flutuação cambial.

Por outro lado, Belluzzo e Carneiro (2004) e Ferrari Filho et al. (2005), por exemplo, apresentam os seguintes problemas dessa estratégia: (i) o fato de que a percepção dos investidores não é dada, podendo ser recebida como falta de confiança no próprio tripé macroeconômico; (ii) a necessidade de manter um volume de reservas em moeda estrangeira ainda maior para sustentar as intervenções no mercado cambial; (iii) a existência de um tradeoff entre os riscos cambial e de conversibilidade, afinal, a abertura da conta capital e financeira amplia a volatilidade cambial, enquanto a manutenção de controles sobre os fluxos de capital cria nos agentes do mercado financeiro a expectativa de uma maior estabilidade da taxa de câmbio no futuro, permitindo a redução do risco cambial; e (iv) a baixa evidência empírica de desdobramentos positivos da abertura e da integração financeira.

Quanto à revisão da literatura empírica, inicialmente é preciso atentar para os diversos indicadores que mensuram o grau de LFE de uma dada economia. Segundo Prasad et al. (2003), há pelo menos dois tipos principais de indicadores que podem ser construídos para capturar tal fenômeno. Os índices de jure ou de abertura financeira apresentam o grau de abertura da conta capital e financeira a partir de uma análise da legislação e da dimensão regulatória, isto é, provêm diretamente das restrições governamentais. Já os índices de facto ou de integração financeira tratam da profundidade dos movimentos de capitais. Para Edison et al. (2002a, 2002b), as duas dimensões da mensuração possuem vantagens e desvantagens, sendo, portanto, complementares. Se, por um lado, as medidas de jure concentram-se nas decisões de regulatórias e de política econômica de um dado país e, dessa maneira, são menos 
sensíveis aos movimentos globais exógenos, por outro, a delimitação de fatores como amplitude, intensidade e efetividade das restrições governamentais responde a avaliações subjetivas dos pesquisadores ${ }^{3}$. Quanto às medidas de facto, a maior objetividade dos dados básicos esbarra em problemas como a multiplicidade de fatores explicativos dos fluxos e a endogeneidade possível entre crescimento e movimento de capitais.

Edison et al. (2002a), Prasad et al. (2003), IMF (2016) e Pagliari e Hannan (2017) apresentam um panorama dos estudos empíricos a respeito dos impactos da LFE sobre diversas variáveis macroeconômicas, em especial, o crescimento do produto. Nesse sentido, destaca-se a ambiguidade dos resultados encontrados em ambas as dimensões. Os diversos indicadores não são significativos em vários exercícios econométricos, tampouco há consenso sobre o sentido de seus desdobramentos. Fluxos de capitais ou sua volatilidade parecem não estar associados a trajetórias robustas e estáveis de crescimento. Por outro lado, há evidências de ampliação da instabilidade, com a emergência recorrente de crises financeiras e piora na distribuição de renda (Furceri e Loungani, 2015; Mirzae et al., 2016).

No caso brasileiro, os estudos dividem-se entre aqueles que avaliam a efetividade dos controles de capital - Cardoso e Goldfajn (1998), Carvalho e Garcia (2008), Chamon e Garcia (2014), Munhoz (2013), dentre outros - e os que focam nos desdobramentos macroeconômicos da liberalização - como Biage et al. (2008), Paula (2011), Faria et al. (2009), Laan (2007), Paula et al. (2012), Cunha e Laan (2013), para citar alguns. Os primeiros apontam que os controles são efetivos, ainda que temporariamente e/ou após um período de tempo. Além disso, desde o artigo seminal de Cardoso e Goldfajn (1998), tais estudos destacam a endogeneidade dos controles de capital, que são ampliados frente a influxos excessivos e reduzidos em períodos de fluxos estáveis ou de saídas de capital.

Já o segundo grupo de trabalhos caracteriza-se pelo emprego de modelos baseados em vetores autorregressivos (VAR-VEC), bem como pela avaliação, em separado, dos efeitos associados a cada dimensão da LFE. Entre as variáveis incluídas nesses exercícios, destacam-se o produto, as reservas oficiais, o risco-país, a taxa de câmbio, a taxa de juros, a volatilidade cambial, dentre outras. Em geral, tais variáveis respondem de forma não significativa ou problemática frente a um avanço no grau tanto de abertura quanto de integração financeira.

Por fim, é possível notar dois aspectos nos quais o presente trabalho pode contribuir com a literatura empírica centrada no caso brasileiro. Em primeiro lugar, ainda que os estudos de Faria et al. (2009), Paula et al. (2012), Cunha e Laan (2013), dentre outros, atentem para o problema das quebras estruturais nas séries temporais utilizadas, nenhum destes o faz a partir do emprego do modelo MS-VAR, o qual permite o tratamento da não linearidade dos parâmetros, aproveitando tais informações para separar as observações em regimes, os quais podem auxiliar na explicação dos desdobramentos verificados. Em segundo lugar, os esforços revisados não procederam a uma análise conjunta dos movimentos do ciclo financeiro global e da liberalização financeira externa brasileira, tal como se pretende no presente artigo.

\footnotetext{
${ }^{3}$ A maior parte dos indicadores referentes ao grau de abertura da conta capital e financeira é derivada do Annual Report on Exchange Arrangements and Exchange Restrictions (AREAER) como o SHARE de Alesina, et al. (1993), o KAOPEN de Chinn e Ito (2008), o OPENESS e o CAPITAL de Quinn (1997), dentre outros.
} 


\section{O processo brasileiro de liberalização financeira externa: histórico e mensuração}

O processo brasileiro de liberalização desconstruiu os pilares da legislação cambial vigente desde o começo dos anos 1930 (Laan et al., 2017): o monopólio do Banco Central na compra de divisas cambiais, as limitações à saída de capitais após o ingresso e as restrições quantitativas em termos de posições cambiais. Segundo Biancarelli (2004), tal processo apresentou três fases: (i) a primeira, que foi até metade da década de 1990, concentrou-se em medidas que facilitaram à entrada de capitais; (ii) a segunda caracteriza-se pela expansão e instalação de instituições financeiras estrangeiras no Brasil; e (iii) a terceira, ocorrida a partir de 1999, com a crise cambial e a instituição do regime cambial flexível, focou-se nos fluxos de saída de capital e em medidas regulatórias complementares, as quais buscavam a redução de exigências burocráticas e restrições operacionais, a elevação da flexibilidade no mercado cambial e a minimização de custos de transação.

Após a crise financeira global de 2007-2008, conferiu-se maior legitimidade à preocupação com a instabilidade decorrente do livre fluxo de capitais, principalmente no que tange à vulnerabilidade financeira de países emergentes (IMF, 2016). Além disso, observa-se uma mudança de situação na administração da conta capital e financeira, afinal, o quadro de escassez de capitais é substituído pelo excesso, colocando a preocupação acerca de questões como a sustentabilidade e a reversão dos fluxos (Laan et al., 2017). Nesse contexto, o emprego do IOF ganha relevância enquanto principal instrumento no controle de capitais. Por exemplo, em outubro de 2009 , introduz-se uma alíquota de $1,5 \%$, logo aumentada para $2 \%$, aplicada a todos os influxos, exceto IED. Essa taxa inviabilizava, economicamente, movimentações de curtíssimo prazo, ao passo que incentivava a migração de investidores estrangeiros para ativos de prazo mais longo. Na mesma direção, foram tomadas outras medidas restritivas como a instituição de uma alíquota de IOF de $6 \%$ sobre empréstimos externos com prazo médio até 360 dias e, em seguida, até 720 dias, bem como o recolhimento compulsório e o encaixe obrigatório sobre posição vendida de câmbio acima de um bilhão de dólares.

A partir de 2012, nota-se uma reversão da tendência restritiva quanto ao IOF em resposta à possibilidade de mudança na condução da política monetária estadunidense, a qual culminaria com uma elevação das taxas de juros do país central, elevando o risco de parada súbita ou saída massiva de capitais investidos em mercados emergentes como o brasileiro. Dessa forma, até o final de 2014, foram tomadas diversas medidas liberalizantes como a eliminação do imposto sobre diversas operações e a flexibilização do recebimento antecipado de pagamentos por exportações de bens e serviços.

Conforme foi exposto na seção anterior, a avaliação dos efeitos da abertura financeira sobre o desempenho macroeconômico aparece de duas formas na literatura empírica: estudos baseados em dados em painel ou concentrados em uma determinada economia nacional. No primeiro grupo, destacam-se os esforços tributários do relatório anual AREAER do FMI, a única fonte de dados internacionais que capta os regimes cambiais nacionais, além de descrever as mudanças normativas anuais. Não obstante, Cunha e Laan (2013) sublinham que tal instrumento é limitado por não considerar as várias intensidades no grau de liberalização, a eficácia das normas existentes e as restrições existentes sobre fluxos específicos. 
Nesse sentido, há diversos estudos que avaliam cada medida regulatória, formando uma série temporal de maior frequência (mensal), porém com foco em uma economia nacional específica. A tradição brasileira, por exemplo, remonta ao trabalho seminal de Cardoso e Goldfajn (1998), que construíram o Índice de Controle de Capitais (ICC). Esse indicador foi utilizado em trabalhos posteriores como Soihet (2002), Laan (2007), Faria et al., (2009), Paula et al. (2012), entre outros.

Tal metodologia concentra-se nas normas editadas pelo Banco Central, pela Receita Federal e governo central, classificando cada normativo em liberalizante, restritivo ou regulamentar. Dado que a atribuição de notas $+1,-1$ ou 0 , respectivamente em cada caso não leva em conta a intensidade das medidas, Cunha e Laan (2013) propõem um novo ICC, incluindo uma gradação entre os normativos ao atribuir notas entre -2 a +2 com intervalos de gradação de .25 , permitindo diferenciar os normativos, liberalizantes ou restritivos. Além disso, o novo ICC emprega uma soma simples das notas concedidas ao longo da amostra, diferentemente da ponderação de Cardoso e Goldfajn (1998), sendo que os valores positivos dizem respeito a decisões regulatórias liberalizantes, enquanto os negativos a medidas restritivas.

Ao se empregar a referida gradação na atribuição de notas, avalia-se o caráter geral de cada normativo, se liberalizante, restritivo ou regulamentar, mas também se procurou quantificar a profundidade, ou não, de cada normativo, considerando estatura legal (tipo de norma), tamanho e alcance de conteúdo. Sublinha-se que o ICC se concentra no objetivo da decisão regulatória e não, no tipo de fluxo afetado. Por outro lado, quanto mais abrangente for a medida regulatória, quanto ao tipo e sentido dos fluxos, maior tende a ser sua nota em termos absolutos.

Há ainda a possibilidade de que a mesma medida combine dispositivos restritivos com liberalizantes. Tal situação foi enfrentada levando em consideração o sentido líquido do normativo. Assim, caso os aspectos restritivos e liberalizantes apresentassem a mesma profundidade, o normativo receberia nota zero. Reconhece-se, em linha com o alerta de Edison et al. (2002a, 2002b), que índices de jure acabam refletindo avaliações subjetivas dos pesquisadores, demandando sua combinação com índices de facto para traçar um panorama coerente sobre o grau de liberalização financeira externa de uma dada economia.

A amostra mensal do ICC entre 1995 e 2014 foi fruto das seguintes fontes: Soihet (2002), que avaliou 103 normas, editadas entre 1995 e 2000; Laan (2007), para mais 84 normativos até setembro de 2005; Cunha e Laan (2013), para 131 regulamentos editados até final de 2011 e para atribuição de notas ponderadas para os normativos analisados por Soihet (2002) e Laan (2007); e consulta direta, em fonte primária, nos sites do Banco Central, Receita Federal e Planalto para outros 43 normativos editados entre 2012 e $2014^{4}$. Sua composição está na tabela 1.

\footnotetext{
${ }^{4}$ Lista dos normativos avaliados: 1995-2000: ver Soihet (2002); 2001-2005T3: ver Laan (2007); 2005T4-2011: ver Cunha e Laan (2013); 2012: Circular 3570, Resolução 4051, Circular 3575, Decreto 7683, Circular 3580, Decreto 7698, Circular 3584, Decreto 7699, Circular 3589, Resolução 4063, Resolução 4071, Resolução 4074, Circular 3592, Decreto 7751, Resolução 4103, Resolução 4104, Circular 3604, Circular 3591, Circular 3605, Resolução 4113, Circular 3607, Decreto 7853, Circular 3612 e Circular 3617. 2013: Decreto 7894, Resolução 4198, Circular 3627, Circular 3650, Circular 3653, Decreto 8023, Decreto 8027, Circular 3661, Circular 3672, Circular 3688, Circular 3689, Circular
} 
Tabela 1. Síntese da legislação (1995-2014)

\begin{tabular}{lll}
\hline Natureza & Quantidade $\%$ \\
\hline Liberalizante & 144 & 39,9 \\
Restritiva & 56 & 15,5 \\
Neutra & 161 & 44,6 \\
Total & 361 & 100,0 \\
\hline
\end{tabular}

Fonte: elaboração própria.

Sublinha-se a importância de atribuir notas proporcionais à intensidade de cada medida avaliada. Por exemplo, o Decreto 7.412/10 recebeu a nota -2 , pois possuiu forte caráter restritivo ao impor novas taxas a diversas categorias de fluxos. Já a Circular 3.607 obteve a nota .25 , pois liberaliza o recebimento e a entrega de moeda via máquinas automáticas no exterior, isto é, afeta um fluxo pouco representativo. Caso fosse seguida a metodologia de Cardoso e Goldfajn (1998), as duas medidas teriam o mesmo peso e sinais opostos, gerando um saldo líquido nulo quando, na verdade, o sentido agregado de ambas fora restritivo.

Após a construção do ICC, segue-se a sugestão de Prasad et al. (2003) e Edison et al. (2002a, 2002b) e se passa à mensuração da dimensão de facto da liberalização financeira externa. Tal procedimento justifica-se pela dificuldade de que apenas um indicador dê conta das duas dimensões do processo de liberalização, isto é, a evolução regulatória e a real integração com os mercados financeiros globalizados.

Quanto à mensuração do grau de integração financeira da economia brasileira, a literatura empírica traz as seguintes alternativas: (i) medida do diferencial de preços no mercado de ativos; (ii) ponderação dos estoques financeiros pelo produto nominal; (iii) ponderação dos fluxos brutos de capital pelo produto nominal; e (iv) consideração do volume dos fluxos financeiros. A primeira alternativa foi empregada por Chamon e Garcia (2014), e remonta aos trabalhos de Obstfeld e Taylor (2004) e Prasad et al. (2003). No entanto, há o risco de tais retornos moverem-se por fatores exógenos, de modo que a redução no diferencial de preços pode refletir choques comuns ao invés de um aumento na integração. Quanto aos estoques, Cunha e Laan (2013) criticam seu emprego por que: (i) pela limitação das séries de tempo, na medida em que para períodos mais longos há apenas dados anuais; (ii) a posição de ativos de brasileiros no exterior (ativos) só passou a ser sistematicamente conhecida a partir de 2005, com a introdução, pelo Banco Central, do Censo Anual de Capitais Brasileiros no Exterior; (iii) exige-se somente a declaração dos capitais brasileiros no exterior acima de USD 100 milhões; e (iv) há imprecisão na quantificação dos ativos estrangeiros.

Com efeito, o emprego de medidas baseadas nos fluxos brutos (soma em módulo de entradas e saídas de capital) mostra-se mais adequado para a realidade brasileira, sendo adotado, portanto, neste artigo. Não obstante, ainda resta tratar da necessidade de ponderação da soma referida pelo produto nominal. Por um lado, Cunha e Laan (2013)

3690, Circular 3691, Decreto 8165 e Decreto 8175. 2014: Circular 3702, Resolução 4335, Decreto 8263, Resolução 4373 e Decreto 8325. 
apontam que a série em dólares divulgada pelo Banco Central é apenas uma transformação direta do produto nominal em reais com a taxa média de dólar para cada mês, de modo que isso prejudicaria o índice por incluir variações relativas ao movimento cambial e não, ao nível de atividade em si. Por outro, considera-se que trabalhar apenas com o volume dos fluxos financeiros não possibilita a avaliação do grau de integração aos mercados financeiros globalizados, isto é, do peso dos fluxos brutos frente à economia em questão. Portanto, a despeito da influência dos movimentos da taxa de câmbio, adota-se um Índice de Integração Financeira (IIF) baseado na ponderação dos fluxos financeiros pelo produto nominal. Nesse ponto, a metodologia apresenta-se em linha os trabalhos de Kraay (1998), Bluedorn et al. (2009), Laan (2007), dentre outros. As contas consideradas seguem a formulação de Cunha e Laan (2013).

\section{Os desdobramentos macroeconômicos da liberalização financeira externa: uma análise a partir do modelo MS-VAR}

Como foi dito anteriormente, a existência de não linearidade nos modelos macroeconômicos também pode ser enfrentada a partir do modelo MS-VAR, o qual remonta aos trabalhos de Krolzig $(1996,1997,1998,2000)$. Sublinha-se também as contribuições de Ehrmann et al.(2003), Kim e Nelson (1999) e Sims e Zha (2004, 2006).

Como contribuição potencialmente original, o emprego, nessa temática, do modelo econométrico de Vetores Autorregressivos com Mudanças Markovianas (MS-VAR) justifica-se a partir dos seguintes pontos, quais sejam: o enfrentamento da não linearidade dos parâmetros com a conservação da endogeneidade das variáveis, sem que isso signifique a imposição de restrições excessivas; e a manutenção do caráter não estacionário de muitas das séries do modelo, evitando a eliminação a priori de informações por meio da diferenciação. Ademais, essa opção permite a utilização de uma abordagem econométrica general to specific, dentro da qual são consideradas diversas relações para que, em exercícios futuros, sejam impostas restrições economicamente significativas.

No que tange ao modelo, as duas primeiras variáveis dizem respeito a diferentes dimensões do grau de liberalização financeira externa da economia brasileira, discutidas na seção anterior e empregando indicadores em linha com a literatura recente sobre o tema. 0 desempenho macroeconômico, por sua vez, pode ser observado a partir do nível de atividade e da taxa de juros, fontes de controvérsia entre os defensores e os críticos da liberalização financeira externa. Além disso, foram incluídas as variáveis risco-país e volatilidade cambial para avaliar a proposta de Arida (2003a, 2003b, 2004) acerca da plena conversibilidade da conta capital.

Ainda sobre a composição da estimação, o ciclo financeiro global é a última variável incluída. Optou-se por empregar o índice de volatilidade VIX como proxy do ciclo, assumindo que momentos de elevada aversão ao risco coincidem com períodos de reversão do ciclo global, enquanto momentos de baixa aversão coincidem com períodos de ascensão cíclica. Reconhece-se que a volatilidade financeira internacional é apenas um entre as diversas 
dimensões do ciclo financeiro global ${ }^{5}$, todavia, diversos estudos como Rey (2013), Nier et al. (2014), IMF (2017), Coimbra e Rey (2017), Cerutti et al. (2017), dentre outros defendem o VIX como proxy do ciclo financeiro global.

O modelo estimado a seguir será, portanto, composto por sete variáveis' as quais não sofreram qualquer tipo de tratamento, conforme orientação de Krolzig (1996, 1997, 1998, 2000). A única exceção refere-se à produção industrial (PROD), dado que esta foi incluída no modelo com o objetivo de representar o nível de atividade. Nesse sentido, as variações sazonais da produção industrial afastariam esta da variável que procura representar. Veja abaixo:

Quadro 1. Especificação das variáveis

\begin{tabular}{|c|c|c|}
\hline Série & Nome & Descrição \\
\hline $\begin{array}{l}\text { Índice de Controle de } \\
\text { Capitais }\end{array}$ & ICCAC & Indicador explicado na seção anterior. \\
\hline $\begin{array}{l}\text { Índice de Integração } \\
\text { Financeira }\end{array}$ & IIF & Indicador explicado na seção anterior. \\
\hline Nível de Atividade & PROD & $\begin{array}{l}\text { Produção industrial - indústria geral - quantum - índice } \\
\text { dessazonalizado (média } 2002=100 \text { ), divulgado na } \\
\text { PIM/IBGE. }\end{array}$ \\
\hline Volatilidade Cambial & CAVOL & $\begin{array}{l}\text { Volatilidade da taxa de câmbio, série BC } 1 \text { - Taxa de } \\
\text { câmbio - Livre - Dólar americano (venda) - diário - } \\
\text { u.m.c./USD, venda fim de período, calculada pelo desvio- } \\
\text { padrão mensal da média diária (Ptax800), divulgada pelo } \\
\text { Depin. }\end{array}$ \\
\hline Taxa de Juros & SELIC & $\begin{array}{l}\text { Série BC } 4189 \text { - Taxa de juros - Selic acumulada no mês } \\
\text { anualizada - \% a.a., divulgada pelo Demab. }\end{array}$ \\
\hline $\begin{array}{l}\text { Ciclo Financeiro } \\
\text { Global }\end{array}$ & VIX & $\begin{array}{l}\text { Índice de Volatilidade, calculado pelo CBOE - média } \\
\text { mensal. }\end{array}$ \\
\hline Risco Brasil & RISCO & $\begin{array}{l}\text { Risco-Brasil, calculado pelo JPMorgan. EMBI+ Risco- Brasil- } \\
\text { média mensal. }\end{array}$ \\
\hline
\end{tabular}

Fonte: Instituto de Pesquisa Econômica Aplicada (IPEA); Banco Central do Brasil (BCB); Chicago Board Options Exchange Market (CBOE).

O modelo MS-VAR é uma evolução do modelo VAR em diversos aspectos. Em modelos de séries temporais, como VAR ou VEC (Vetor de Correção de Erros), a identificação começa com a avaliação da estacionaridade das séries. Todavia, há uma ampla discussão sobre a especificação de um modelo VAR. Segundo Sims (1990), as séries não devem ser diferenciadas, dado que o objetivo da estimação é compreender as relações entre as variáveis, e a diferenciação leva à perda destas. Nesse sentido, Bernanke e Mihov (1998) afirmam que a

\footnotetext{
${ }^{5}$ Autores como Bruno e Shin (2012) e Borio e Disyatat (2011), por exemplo, empregam os influxos de capitais ou empréstimos do setor bancário entre fronteiras para mensurar a liquidez global.
} 
estimação do modelo VAR com as variáveis em nível produz estimadores estatisticamente consistentes, independentemente da existência de vetores de cointegração.

Dito isto, opta-se por estimar um modelo MS-VAR com variáveis em nível. Considerando os critérios de informação de Akaike, Schawrz e Hannan-Quinn, estimou-se um modelo com uma defasagem (ver testes no Anexo). O regime 1 diz respeito a momentos de alto nível de volatilidade financeira internacional (mensurada pelo VIX) e, portanto, reversão do ciclo financeiro global. Já o regime 2 é composto pelos períodos de expansão do ciclo financeiro global e, consequentemente, um baixo nível de volatilidade nos mercados financeiros mundiais. Os dados são usados para estimar e analisar um modelo MS-VAR irrestrito, com intercepto, variância e parâmetros variando conforme o regime. Dessa forma, estimou-se um MS(2)-VAR(1) ou, conforme Krolzig (1997), um MSIAH(2)-VAR(1). O número de parâmetros estimados foi 121, inferior às 240 observações da amostra.

A justificativa para o emprego do modelo MS-VAR assenta-se no caráter não linear por parte dos parâmetros, isto é, na mudança significativa destes entre os regimes. Indo ao encontro disso, o Teste LR de linearidade rejeitou a hipótese nula de linearidade com um grau de confiança de $99 \%$. A convergência do algoritmo EM deu-se após quatro interações, com uma probabilidade de mudança de 0,0001. 0 gráfico 1 mostra o bom ajustamento do modelo em cada regime estimado.

Gráfico 1. Ajustamento do modelo MS(2)-VAR(1) aos regimes (1995/2014)

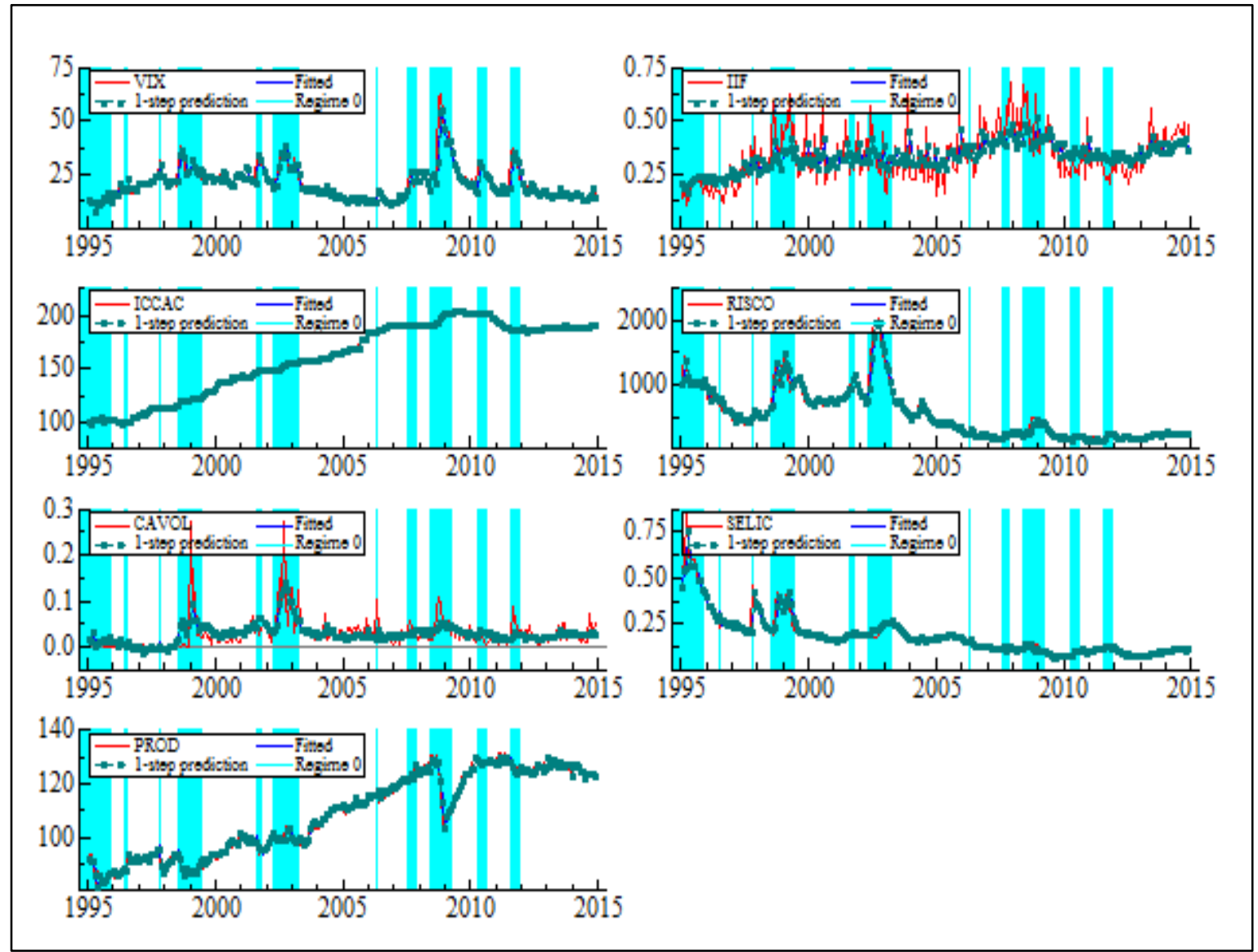

Fonte: elaboração própria. 
Nota-se que o regime 1 caracteriza-se pela coincidência entre níveis mais elevados para o grau de integração financeira, o risco-país, a taxa de juros e a volatilidade cambial. 0 modelo estimado mostrou uma matriz de transição com regimes persistentes, isto é, uma vez que se está em um dos regimes, a probabilidade de se conservar neste é alta. Segundo tal matriz, estando no primeiro regime, a probabilidade de mudar para o segundo regime é de apenas $7,7677 \%$, enquanto que, para permanecer nele, a probabilidade é de $92,232 \%$. 0 mesmo ocorre no segundo regime, uma vez estando nele, a probabilidade de mudança é de apenas $4,25 \%$, enquanto a de permanência é de $95,75 \%$. De acordo com as probabilidades estimadas, os regimes podem ser classificados temporalmente (tabela 2). 0 regime dois mostra-se mais persistente, totalizando 178 meses do período analisado e tendo uma média de duração de aproximadamente 20 meses. Já o regime um totaliza apenas 60 meses, tendo uma média de 6 meses de duração.

Tabela 2. Classificação estimada dos regimes do modelo MS(2)-VAR(1)

\begin{tabular}{ll}
\hline Regime 1 & Regime 2 \\
\hline $02 / 1995-11 / 1995(0,960)$ & $12 / 1995-06 / 1996(0,813)$ \\
$07 / 1996-07 / 1996(0,869)$ & $08 / 1996-10 / 1996(0,998)$ \\
$11 / 1997-11 / 1997(1,000)$ & $12 / 1997-07 / 1998(0,986)$ \\
$08 / 1998-06 / 1999(0,963)$ & $07 / 1999-08 / 2001(0,998)$ \\
$09 / 2001$ - 10/2001 $(0,936)$ & $11 / 2001-04 / 2002(0,991)$ \\
$05 / 2002-04 / 2003(0,973)$ & $05 / 2003-04 / 2006(0,996)$ \\
$05 / 2006-05 / 2006(1,000)$ & $06 / 2006-07 / 2007(0,998)$ \\
$08 / 2007-11 / 2007(0,771)$ & $12 / 2007-05 / 2008(0,792)$ \\
$06 / 2008$ - 04/2009 $(0,965)$ & $05 / 2009-04 / 2010(0,941)$ \\
$05 / 2010-08 / 2010(0,876)$ & $09 / 2010-07 / 2011(0,947)$ \\
$08 / 2011$ - 12/2011 $(0,955)$ & $01 / 2012-12 / 2014(0,997)$ \\
\hline Total: 62 meses & $\begin{array}{l}\text { Total: } 177 \text { meses } \\
74,06 \% \text { do período estimado com } \\
\text { uma média de duração de } 16,09\end{array}$ \\
\hline $\begin{array}{l}\text { meses. } \\
\text { duração de 5,64 meses. }\end{array}$ & \\
\hline
\end{tabular}

Fonte: elaboração própria. A probabilidade está entre parêntesis.

Para analisar as relações entre as variáveis endógenas dentro do modelo MS-VAR, usualmente, constrói-se funções de resposta a impulsos generalizados, conforme o gráfico 4, onde aparecem os regimes 1 (em vermelho) e 2 (em azul). 
Gráfico 3. Funções de Resposta ao Impulso Dependentes dos Regimes 1 e $2^{6}$

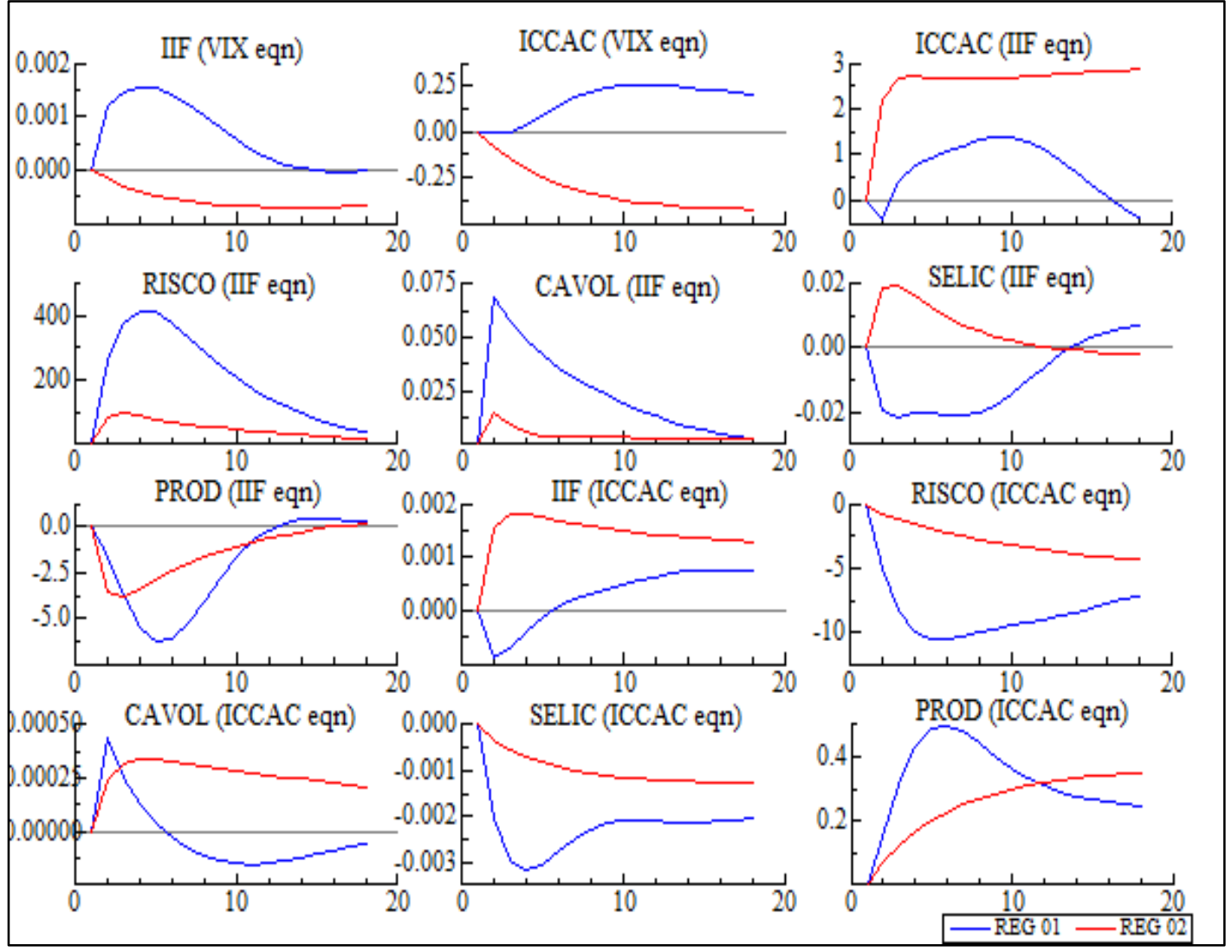

Fonte: elaboração própria.

O primeiro ponto a ser destacado é o impacto do ciclo financeiro global (VIX) sobre as duas dimensões da LFE da economia brasileira, as quais reagem a aumento da volatilidade financeira global de forma contingente ao estágio vigente do ciclo financeiro. Em períodos de reversão do ciclo global, um aumento da volatilidade engendra um avanço do grau de integração (IIF). Isso se dá sob a forma de saídas de capital. As variações do grau de abertura (ICCAC) emergem para flexibilizar as restrições, com o aparente intuito de mitigar esse processo. No regime caracterizado pela ascensão cíclica, a resposta é inversa, principalmente, quanto ao grau de abertura, que é reduzido frente ao ambiente de maior liquidez internacional. Isso acrescenta informação à tese da endogeneidade de Cardoso e Goldfajn (1998), dado que os controles de capital respondem não só a fluxos excessivos de capital, mas também a aumentos da incerteza nos mercados financeiros globais.

Quanto à relação entre os dois indicadores da liberalização, nota-se que as decisões jurídicas e regulatórias apresentam um sentido diferente dependendo do regime no qual se encontra. Dessa maneira, um choque positivo no IIF durante o período de ascensão do ciclo financeiro global é respondido com medidas liberalizantes e, consequentemente, um aumento no ICCAC. Todavia, quando se atravessa um período de reversão cíclica, aumentos conjunturais no grau de integração financeira da economia brasileira engendram respostas restritivas, ao menos em um primeiro momento, em termos de controles de capital. Invertendo a relação,

\footnotetext{
${ }^{6}$ Choque de um ponto percentual em VIX com resposta em IIF; e choques de um ponto percentual em IIF e ICCAC com resposta nas outras variáveis, exceto VIX.
} 
um choque de medidas liberalizantes, isto é, um aumento no ICCAC eleva o grau de integração financeira em quaisquer dos regimes. No entanto, em períodos de maior volatilidade financeira global, há uma reação negativa por parte do IIF nos meses iniciais. Tal especificidade apenas reforça o caráter autônomo dos fluxos de capital, de modo que em situações de crise, estes "fogem para a qualidade", abandonando mercados emergentes como o brasileiro a despeito de seus "fundamentos" econômicos e institucionais.

Os efeitos de um aumento no grau de integração financeira podem ser separados em dois grupos. Em primeiro lugar, um choque no IIF engendra consequências claramente negativas em termos de nível de atividade (redução da produção industrial), risco-país (elevação) e volatilidade cambial (idem). Como esperado, os desdobramentos são mais profundos no regime 1 devido à pressão advinda do ambiente financeiro internacional. Quanto à taxa de juros, o primeiro aspecto a destacar é o aumento do impacto do grau de integração financeira da economia durante períodos caracterizados por uma maior instabilidade financeira internacional. Ademais, um choque positivo no IIF durante períodos de reversão cíclica está associado a uma redução da taxa de juros. Já em um contexto de menor volatilidade, nota-se um aumento da taxa. De qualquer forma, mudanças no grau de integração financeira da economia brasileira afetam a autonomia da política monetária.

Os desdobramentos problemáticos associados a aumento do grau de integração financeira da economia brasileira vão de encontro à defesa da liberalização financeira externa enquanto componente de uma estratégia bem-sucedida de crescimento. Nesse sentido, os resultados corroboram o ceticismo da tradição pós-keynesiana com esse tipo de política, discutindo em seções anteriores.

Para as funções derivadas de um choque na variável ICCAC, o primeiro ponto a ser sublinhado diz respeito ao caráter pouco significativo dos desdobramentos macroeconômicos observados. Nesse sentido, em que pese ter havido, em ambos os regimes, uma elevação do nível de atividade e da oferta de crédito, bem como uma queda no risco-país e na taxa de juros, a magnitude das reações é muito pequena em termos absolutos, principalmente se comparada às reações a choques no IIF. Esses resultados não corroboram a hipótese de Arida (2003a, 2003b, 2004), a qual relaciona a conversibilidade plena da conta capital e financeira a consequências macroeconômicas robustas, em especial, quanto à taxa de juros e ao risco país. Vale notar também que, como um choque positivo no ICCAC leva a uma elevação do IIF nos dois regimes, o pequeno saldo positivo de medidas liberalizantes, se significativo fosse, engendraria consequências negativas, de intensidade superior, ao promover um aumento no grau de integração financeira da economia em questão.

\section{Considerações finais}

A crise financeira global e o ambiente da "Grande Recessão" produziram uma renovação no debate acadêmico e normativo sobre os efeitos da liberalização financeira e as melhores estratégicas para a estabilização macroeconômica e financeira. Economistas, formuladores de política econômica e operadores financeiros, até então defensores do modelo de liberalização financeira irrestrita e de desregulamentação dos diversos mercados de produtos e fatores, vêm matizando suas posições em nome de um intervencionismo pragmático. Passou-se a defender, de forma cada vez mais explícita, políticas até 
recentemente consideradas não convencionais, de recorte contracíclico, bem como maior regulação sobre os mercados financeiros (IMF, 2016).

Foi neste contexto que o presente trabalho procurou resgatar e avançar sobre a literatura prévia ${ }^{7}$, especialmente dos trabalhos construídos na tradição pós-keynesiana, que investigou os efeitos da liberalização financeira externa na economia brasileira por meio da construção de indicadores de jure e de facto. Mais especificamente, nossa contribuição está: (i) na utilização do modelo econométrico de Vetores Autorregressivos com Mudanças Markovianas (MS-VAR), o que ainda não havia sido objeto de escolha metodológica em pesquisas sobre esse tema que nos precederam; (ii) na discussão do impacto de um choque na volatilidade financeira internacional sobre a evolução do grau de liberalização financeira externa da economia brasileira.

Quanto aos resultados, estes, vão ao encontro da preocupação pós-keynesiana, de autores como Belluzzo e Carneiro (2004), Ferrari-Filho et al. (2005), Oreiro (2004), Paula et al. (2012), dentre outros, com as consequências da estratégia de abertura e integração financeira, preconizada por Arida (2003a, 2003b, 2004). Em termos de metodologia econométrica, o modelo MS-VAR acrescenta informações ao permitir o tratamento do caráter não linear dos dados sem recorrer restrições excessivas, bem como ao lançar luz nas consequências diferentes da liberalização financeira externa em regimes caracterizados por uma maior estabilidade ou instabilidade no estado de expectativas dos mercados financeiros globalizados. Quanto ao debate teórico, os resultados são convergentes à posição crítica à liberalização devido aos seguintes aspectos: i) a resposta dos graus de abertura e integração financeira aos movimentos do ciclo financeiro global; ii) os efeitos pouco significativos de medidas regulatórias liberalizantes; e iii) os desdobramentos macroeconômicos problemáticos associados aos choques positivos no grau de integração da economia brasileira aos mercados financeiros internacionais.

Ao concluir este artigo, vale à pena retomar alguns elementos. Em primeiro lugar, ressalta-se que a liberalização financeira de facto e de jure ainda é um tema controverso em termos teóricos e empíricos. Dessa maneira, quando se avalia a correção de medidas nesse sentido, no contexto de uma economia que não faz parte dos mercados avançados e não dispõe de moeda conversível, há que se adotar uma perspectiva pragmática, que leve em conta o ciclo financeiro global, e a posição de tomadora deste por parte da economia brasileira. A observação de um impacto relevante de um choque no VIX sobre o grau de liberalização financeira da economia brasileira parece legitimar tal abordagem. Nesse sentido, os resultados do presente artigo apontam a necessidade do emprego de controles de capital, os quais devem combinar uma dimensão permanente, para mitigar os efeitos negativos da integração financeira, com uma dimensão cíclica, voltada para as pressões engendradas pelo ciclo financeiro global.

\footnotetext{
${ }^{7}$ Especialmente, mas não somente, Belluzzo e Carneiro (2004), Ferrari et al. (2005), Laan (2007), Arestis e Paula (2008), Paula (2011) e Paula et al. (2012). Na perspectiva convencional ver, dentre outros, Cardoso e Goldfajn (1998), Arida (2003a, 2003b, 2004) e Chamon e Garcia (2014).
} 


\section{Referências}

Alesina, A.; Grilli, V. e Milesi-Ferretti, G. (1993) "The political economy of capital controls", NBER Working Paper Series, Cambridge (MA), n. 4353.

Arestis, P.; Sobreira, R. e Oreiro, J. (2011) “An Assessment of the Global Impact of the Financial Crisis", Houndmills, Basingstoke: Palgrave Macmillan.

Arestis, P. e Paula, L. (2008) "Introduction", In: P. Arestis e L. Paula (Orgs.). Financial Liberalization and Economic Performance in Emerging Countries. Basingstoke: Palgrave Macmillan.

Arida, P. (2003a) “Ainda a conversibilidade", Revista de Economia Política, São Paulo, 23(3), p. 135-142.

Arida, P. (2003b) "Por uma moeda plenamente conversível", Revista de Economia Política, 23(3), p. 151-154.

Arida, P. (2004) "Aspectos macroeconômicos da conversibilidade: uma discussão do caso brasileiro", Disponível em: http://www.econ.pucrio.br/gfranco/Conversibilidade.Arida.25.02.pdf. Acesso em 15/03/2015.

Balassa, B. (1989) "Financial Liberalization in Developing Countries", World Bank Working Paper, Washington D. C., n. 55.

Belluzzo, L. e Carneiro, R. (2004) "O mito da conversibilidade", Revista de Economia Política, 24(2), p. 218-222.

Bernanke, B. e Mihov, I. (1998) "Measuring monetary policy", The Quarterly Journal of Economics, 113(3), p. 869-902.

Biage, M.; Corrêa, V. e Neder, H. (2008) "Risco País, fluxos de capitais e determinação da taxa de juros no Brasil: uma análise de impactos por meio da metodologia VEC", Revista de Economia, Brasília, 9, p. 110-150.

Biancarelli, A. (2004) "Liberalização financeira e política econômica no Brasil recente: três momentos e duas visões", In: Anais do IX Encontro Nacional de Economia Política, Uberlândia: SEP.

BIS (2014) 84th Annual Report. Basileia: Bank for International Settlements.

BIS (2015) 85th Annual Report. Basileia: Bank for International Settlements.

Bluedorn, J.; Duttagupta, R.; Guajardo, J. e Topalova, P. (2009) "Capital flows are fickle: anytime, anywhere", IMF Working Paper, Washington D. C., n. 183.

Borio, C. (2012) "The financial cycle and macroeconomics: What have we learnt?" BIS Working Paper, Basle, n. 395.

Borio, C. e Disyatat, P. (2011) "Global imbalances and the financial crisis: Link or no link?", BIS Working Paper, Basle, n. 346.

Bruno, V. e Shin. H. (2012) "Capital Flows, Cross-Border Banking and Global Liquidity", Working paper, Princeton University.

Cardoso, E. e Goldfajn, I. (1998) "Capital Flows to Brazil: endogeneity of capital controls", IMF Staff Papers, 45(1), p. 161-202.

Carvalho, B. e Garcia, M. (2008) "Ineffective controls on capital inflows under sophisticated financial markets: Brazil in the nineties", In: S. Edwards e M. Garcia (Orgs.) Financial 
markets volatility and performance in emerging markets. Chicago: University of Chicago Press.

Cerutti, E.; Claessens, S. e Rose, A. (2017) "How Important is the Global Financial Cycle? Evidence from Capital Flows", IMF Working Paper, m. 17/193, September. Washington, DC: International Monetary Fund.

Chamon, M. e Garcia, M. (2014) "Capital controls in Brazil: effective?", Paper presented at the 15th Jacques Polak annual conference, Washington D. C.

Chin, M. e Ito, H. (2008) "A New Measure of Financial Openness", Journal of Comparative Policy Analysis, 10, p. 309-22.

Coimbra, N. e Rey, H. (2017) "Financial Cycles with Heterogeneous Intermediaries", NBER Working Paper, n. 23245, March. Cambridge, MA: National Bureau of Economic Research.

Crotty, J. (1983) "On Keynes and capital flight", Journal of Economic Literature, v. 21, n. 1, p. 59-65.

Cynamon, B.; Fazzari, S. e Setterfield, M. (2013) After the Great Recession: the Struggle for Economic Recovery and Growth, Cambridge University Press.

Cunha, A. M.; Laan, C. R. V. D. (2013) "Uma nova ordem financeira internacional? Avaliando alternativas para o Brasil", Textos para Discussão FCE/UFRGS, n. 5, 2013. (Texto para Discussão, p. 1-109.

Davidson, P. (2015) Post Keynesian Theory and Policy: A Realistic Analysis of the Market Oriented Capitalist Economy, Cheltenham: Edward Elgar Publishing, 2015.

Edison, H.; Klein, M.; Ricci, L. e Slok, T. (2002a) "Capital Account Liberalization and Economic Performance: a review of the literature", IMF Working Paper, Washington D. C., n. 120.

Edison, H.; Levine, R.; Ricci, L. e Slok, T. (2002b) "International Financial Integration and Economic Growth", Journal of International Money and Finance, 21(6), p. 749-76.

Ehrmann, M.; Ellison, M. e Valla, N. (2003) "Regime-dependent impulse response functions in a Markov-switching vector autoregression model", Economics Letters, 78(3), p. 295-299.

Faria, J.; Paula, L.; Meyer, T. e Pires, M. (2009) "Financial liberalization, economic performance and macroeconomic stability in Brazil: an assessment of the recent period", Anais do 370 Encontro Nacional de Economia da ANPEC, Foz do Iguaçu: ANPEC.

Ferrari Filho, F.; Jayme Jr., F.; Lima, G.; Oreiro, J. e Paula, L. (2005) "Uma avaliação crítica da proposta de conversibilidade plena do Real", Revista de Economia Política, 25(1), p. 133151.

Fischer, S. (1998) "Capital account liberalization and the role of IMF. Essays in International Finance", Princeton, n. 207, p. 1-11.

Furceri, D. e Loungani, P. (2015) "Capital Account Liberalization and Inequality", IMF Working Paper, 15(243), Washington, DC: IMF.

Gallagher, K. (2012) "The Myth of Financial Protectionism: The New (and Old) Economics of Capital Controls", PERI Working Paper, Amherst, n. 278.

Henry, P. (2007) "Capital account liberalization: theory, evidences, and speculation", Journal of Economic Literature, Pittsburgh, n. 45, p. 887-935.

IMF (2016) "Capital Flows: Review of Experience with the Institutional View", Washington, DC, International Monetary Fund. 
IMF (2017) "Global Financial Stability Report: Getting the Policy Mix Right", Washington, DC, International Monetary Fund.

Keynes, J. M. (1976) A Treatise on Money, New York: AMS Press.

Kim, C. e Nelson, C. (1999) State-space models with regime switching: classical and Gibbssampling approaches with applications, MIT Press Books, v. 1.

Korinek, A. (2011) "The New Economics of Capital Controls: Imposed for Prudential Reasons", IMF Working Paper, 298(11), Washington, DC: IMF, 2011.

Kraay, A. (1998) In Search of Macroeconomic Effects of Capital Account Liberalization, Washington D.C.: World Bank.

Kregel, J. (1996) "Riscos e implicações da globalização financeira para a autonomia de políticas nacionais", Economia e Sociedade, Campinas, 7, p. 29-49.

Kregel, J. (2004) "External financing for development and international financial instability", UNCTAD G-24 Discussion Paper Series, n. 32.

Krolzig, H. (1996) "Statistical analysis of cointegrated VAR processes with Markovian regime shifts", SFB 373 Discussion Paper, Berlin, n. 25.

Krolzig, H. (1997) Markov-switching vector autoregressions: Modelling, statistical inference, and application to business cycle analysis, Berlin: Springer.

Krolzig, H. (1998) "Econometric modelling of Markov-switching vector autoregressions using MSVAR for Ox", Mimeo.

Krolzig, H. (2000) "Predicting Markov-switching vector autoregressive processes", Oxford Discussion Paper, n.31, Oxford.

Lavoie, M. (2014) Post-Keynesian Economics: New Foundations, Edward Elgar, 2014.

Laan, C. (2007) Liberalização da Conta de Capitais: Evolução e Evidências para o Caso Brasileiro Recente (1990-2005), Rio de Janeiro: BNDES.

Laan, C.; Cunha, A. M.; Lélis, M. T. C. (2017) "On the effectiveness of capital controls during the Great Recession: The Brazilian experience (2007-2013)", Journal of Post Keynesian Economics, 40(2), p. 203-222.

Levine, R. (2001) "International finance liberalization and economic growth", Review of International Economics, 9(4), p. 688-702.

Mckinnon, R. (1973) Money and Capital in Economic Development, Washington, DC: Brookings Institution.

Minsky, H. P. (1993) "Financial Integration and National Economic Policy", Hyman P. Minsky Archive. Paper 41, Disponível em: http://digitalcommons.bard.edu/hm_archive/41/. Acesso em 15/03/2018.

Mirzae, A.; Kutan, A. e Igan, D. (2016) "Real Effects of Capital Inflows in Emerging Markets", IMF Working Paper, n. 16/235. Washington, DC: International Monetary Fund.

Mishkin, F. (2005) "Is Financial Globalization Beneficial?" NBER Working Paper, Cambridge (MA), n. 11891.

Munhoz, V. (2013) "Vulnerabilidade externa e controle de capitais no Brasil: uma análise das inter-relações entre câmbio, fluxos de capitais, IOF, juros e risco-país", Nova Economia, Belo Horizonte, 23 (2), p. 371-402. 
Nier, E.; Sedik, T. e Mondino, T. (2014), "Gross Private Capital Flows to Emerging Markets: Can the Global Financial Cycle Be Tamed?", IMF Working Paper, Washington D. C., n. 196.

Obstfeld, M. e Taylor, A. (2004) Global capital markets: integration, crisis and growth, New York: Cambridge University Press.

Ocampo, J.; Kregel, J. e Griffith-Jones, S. (2007) International Finance and Development, London and New York: Zed Books.

Oreiro, J. (2004) “Autonomia de política econômica, fragilidade externa e equilíbrio do balanço de pagamentos: a teoria econômica dos controles de capitais", Economia e Sociedade, 13(2), p. 1-22.

Palley, T. (2009) "Rethinking the economics of capital mobility and capital controls", Revista de Economia Política, 29(3) (115), p. 15-34.

Palley, T. (2013) From Financial Crisis to Stagnation: The Destruction of Shared Prosperity and the Role of Economics. Cambridge: Cambridge University Press.

Pagliari, M. e Hannan, S. (2017) "The Volatility of Capital Flows in Emerging Markets: Measures and Determinants", IMF Working Paper, WP/17/41. Washington, DC: International Monetary Fund.

Paula, L. (2011) Financial Liberalization and Economic Performance: Brazil at the crossroads, London: Routledge.

Paula, L.; Pires, M.; Faria Junior, J. e Meyer, T. (2012) "Liberalização financeira, performance econômica e estabilidade macroeconômica no Brasil: uma análise do período 1994-2007", Nova Economia, Belo Horizonte, 22(3), p. 561-596.

Paula, L.; Fritz, B. e Prates, D. (2017) "Keynes at the Periphery: Currency hierarchy and challenges for economic policy in emerging economies", Journal of Post Keynesian Economics, 40(2), p. 183-202.

Prasad, E.; Rogoff, K.; Wei, S. e Kose, M. (2003) Effects of Financial Globalization on Developing Countries: Some Empirical Evidence, Washington, DC: International Monetary Fund.

Prasad, E.; Rajan, R. e Subramanian, A. (2007) "Foreign capital and economic growth", IZA Discussion Papers, Bonn, n. 3186.

Quinn, D. (1997) "The correlates of changes in international financial regulation", American Political Science Review, Cambridge, 91(3), p. 531-551.

Rey, H. (2013) "Dilemma not Trilemma: The global financial cycle and monetary policy independence", 25th Jackson Hole symposium, Kansas City.

Shaw, E. (1973) Financial Deepening in Economic Development, New York: Oxford University Press.

Sims, C. (1990) Macroeconomics and reality. Modelling Economic Series, Oxford: Clarendon Press.

Sims, C. e Zha, T. (2004) "MCMC method for Markov mixture simultaneous-equation models: a note", Atlanta: Federal Reserve Bank of Atlanta.

Sims, C. e Zha, T. (2006) "Were there regime switches in US monetary policy?", The American Economic Review, 96(1), Nashville, p. 54-81.

Soihet, E. (2002) "Índice de controle de capitais: uma análise da legislação e dos determinantes do fluxo de capital no Brasil no período 1990-2000", Dissertação (Mestrado em Economia) - Fundação Getúlio Vargas, Rio de Janeiro. 
Stiglitz, J. (2000) "Capital market liberalization, economic growth, and instability", World Development, Washington D. C., 28(6), p. 1075-1086.

Thirlwall, A. (2005) A Natureza do Crescimento Econômico: um referencial alternativo para compreender o desempenho das nações, Brasília, IPEA.

\section{Anexos}

Tabela A1. Teste de seleção de defasagem

\begin{tabular}{ccccccc}
\hline Lag & LogL & LR & FPE & AIC & SC & HQ \\
\hline 1 & $-1664,969$ & 3275,266 & 0,004503 & 14,46192 & $\mathbf{1 5 , 2 7 8 9 3 *}$ & $\mathbf{1 4 , 7 9 1 1 9 *}$ \\
2 & $-1582,282$ & $\mathbf{1 5 4 , 9 5 1 9 *}$ & $\mathbf{0 , 0 0 3 3 9 6 *}$ & $\mathbf{1 4 , 1 7 8 8 4 *}$ & 15,71072 & 14,79621
\end{tabular}

Fonte: Elaboração própria. ${ }^{*}$ Indica o número de defasagens selecionado por cada critério para o sistema VAR. LR: estatística LR; FPE: erro final de previsão; AIC: critério de informação de Akaike; SC: critério de informação de Schwarz; HQ: critério de informação de Hannan-Quinn.

Figura A2. Comportamento dos resíduos no MS(2)-VAR(1)

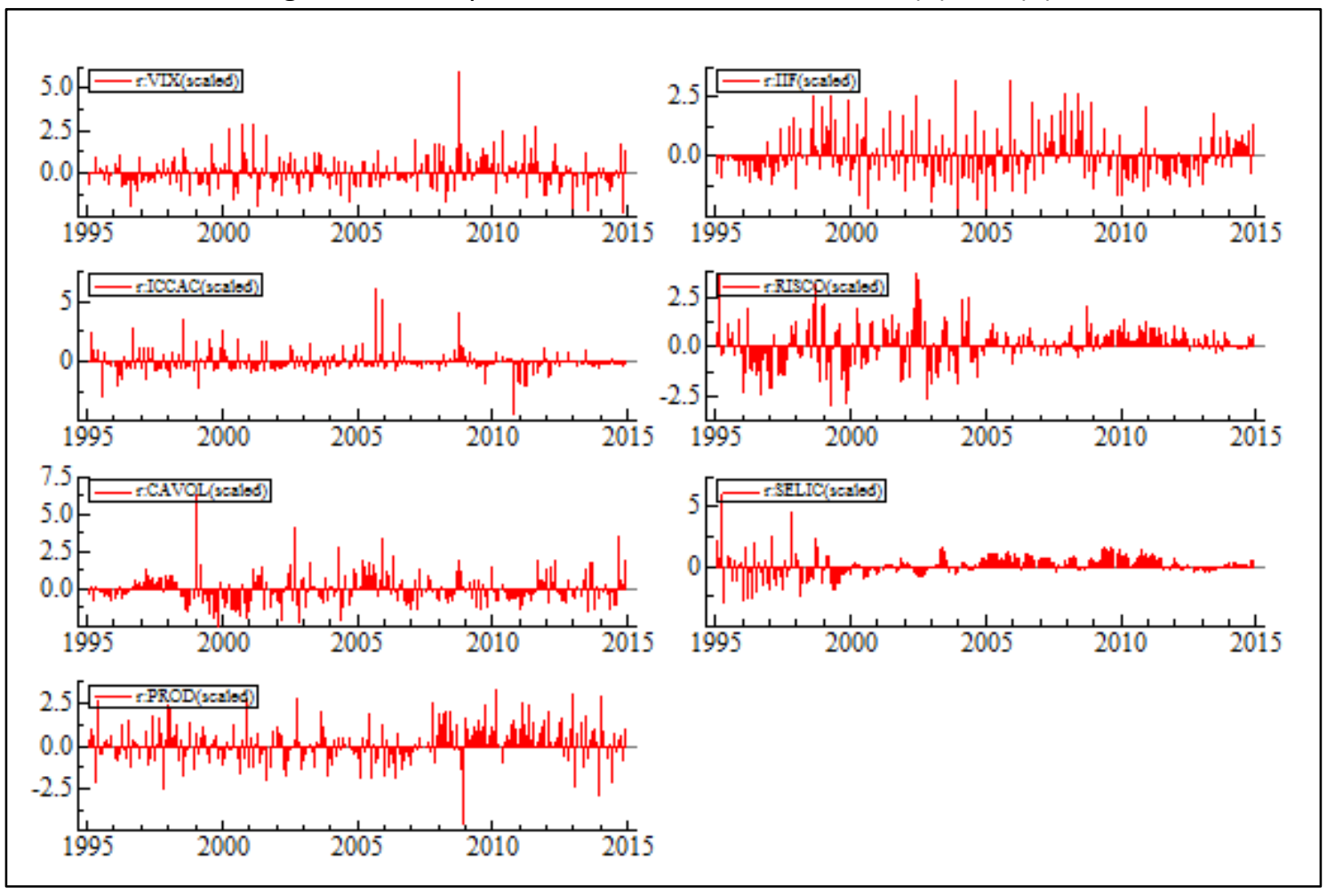

Fonte: saída do software OxMetrics 7.0. Elaboração própria. 
Figura A3. Correlograma, densidade e QQ-Plot dos resíduos no MS(2)-VAR(1)

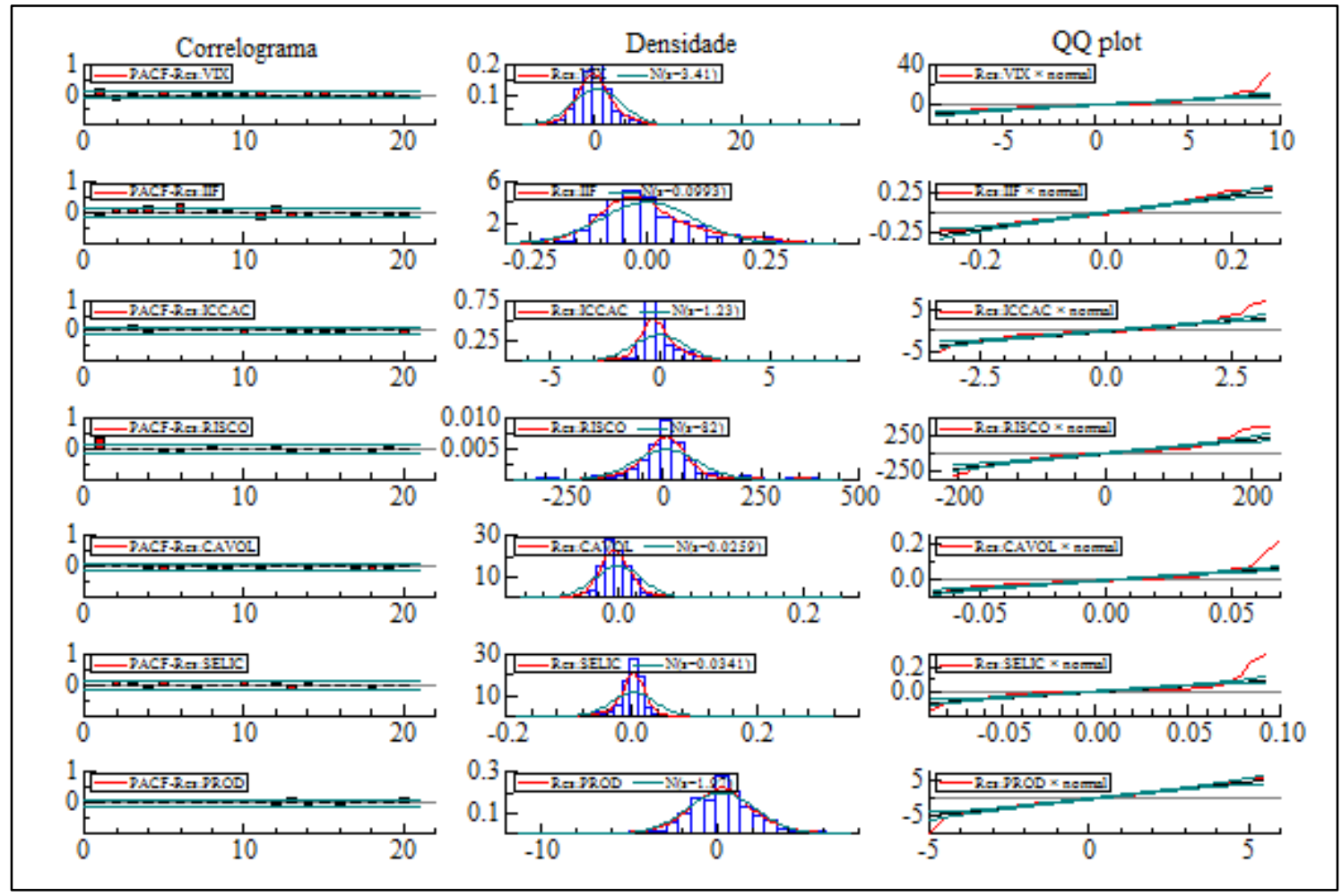

Fonte: saídas do software OxMetrics 7.0. Elaboração própria. 